\title{
Avaliação dos indicadores de aquisição, disponibilidade e adequação nutricional da cesta básica de alimentos brasileira
}

\author{
Assessment of the indicators of purchasing, availability, and \\ nutritional adequacy of the Brazilian basic food basket
}

André Bento Chaves Santana (https://orcid.org/0000-0003-4237-3924) ${ }^{1}$

Flávia Mori Sarti (https://orcid.org/0000-0003-2834-2005) ${ }^{2}$

${ }^{1}$ Instituto de Saúde e Biotecnologia, Universidade Federal do Amazonas. Estrada Coari-Mamiá 305, Coari. 69460-000 Manaus AM Brasil.

ibentoi@gmail.com

${ }^{2}$ Escola de Artes, Ciências e

Humanidades, Universidade de São Paulo. São Paulo SP Brasil.

\begin{abstract}
The scope of this study was to assess the evolution of costs for purchasing the basic food basket associated with the purchasing power of the minimum wage, besides measuring the availability per capita in Brazilian households of the items comprised in the food basket. Additional$l y$, adequacy of the nutritional composition and diet quality based on daily consumption of food basket items was evaluated. The results obtained indicate the existence of periods of price increase for the acquisition of the food basket items, as well as the inability of the minimum wage to meet the cost of such food supplies. It was verified that the diets based on the consumption of food basket items have a higher calorie and sodium content than the recommendations for adults, as well as insufficient amounts of calcium, potassium, and vitamin $A$. The evaluation of the quality of the diets indicated low amounts of fruit, vegetables, cereals in the diet consisting of the items of the food baskets, with excess calories from fats and added sugar being verified. In view of such evidence, a review of the composition of the food baskets is recommended in order to meet basic nutritional requirements in food for the Brazilian population. Key words Food basket, Food purchase, Nutritional composition
\end{abstract}

Resumo O objetivo deste estudo foi avaliar a evolução dos custos para aquisição da cesta básica associados ao poder aquisitivo do salário mínimo, além de mensurar a disponibilidade per capita nos domicílios brasileiros dos alimentos que compõem a cesta básica. Também foram avaliadas a adequação da composição nutricional e a qualidade da dieta baseada no consumo diário de itens da cesta básica. Os resultados obtidos apontam a existência de períodos de elevação de preços para aquisição dos itens da cesta básica, assim como a incapacidade do salário mínimo para o custeio para tais provisões alimentares. Foi verificado que as dietas baseadas no consumo dos itens da cesta básica apresentam quantidade de energia e sódio superiores às recomendações para indivíduos adultos, além de apresentar quantidades insuficientes de cálcio, potássio e vitamina $A$. A avaliação da qualidade das dietas apontou baixa quantidade de frutas, vegetais, cereais na dieta composta pelos itens das cestas básicas, sendo constatado excesso energético proveniente de gorduras e açúcar de adição. Diante de tais evidências, recomendase revisão da composição das cestas básicas para atendimento de requisitos nutricionais básicos em alimentação para população brasileira.

Palavras-chave Cesta básica de alimentos, Aquisição de alimentos, Composição nutricional 


\section{Introdução}

A temática das condições de vida e sua influência sobre situação da alimentação no Brasil emerge como objeto de estudo em meados da década de 1930, em decorrência da atuação de Josué de Castro na investigação do problema da fome entre trabalhadores ${ }^{1}$. A partir da publicação de Condições de vida das classes operárias do Recife em 1933, identificou-se a fome crônica por falta de condições para aquisição de alimentos como principal causa da baixa produtividade e alto absenteísmo entre operários, resultando em um ciclo vicioso de fome, doença e pobreza devido às condições de vida precárias².

O estudo de Josué de Castro constituiu um marco para investigação dos determinantes sociais da saúde no país, a partir da dimensão nutricional, contribuindo ao debate sobre papel da alimentação na formação de capital humano para desenvolvimento socioeconômico no Brasil, assim como ao impulso nas políticas públicas de alimentação e nutrição, inclusive na proposição de níveis mínimos de remuneração aos trabalhadores e iniciativas em prol da formação de profissionais em nível superior e investimento em pesquisa científica na área ${ }^{1,3}$.

Destaca-se adicionalmente que este trabalho constituiu o primeiro inquérito de condições de vida e alimentação de classes trabalhadoras conduzido no país, servindo de base para regulamentação do Decreto-lei 299 de 30 de abril de 1938, que instituiu "Comissões do Salário Mínimo" e definiu uma "ração-tipo essencial mínima", base de provisões mínimas para garantia da alimentação dos trabalhadores ${ }^{4}$. Os parâmetros de composição da cesta básica de alimentos necessária para subsistência do trabalhador e proporção da renda necessária para custeio da alimentação de um trabalhador foram instituídos em diferentes níveis, de acordo com distintas regiões do Brasil.

Atualmente, a ração-tipo essencial mínima ainda prevalece para composição da cesta básica de alimentos, sem qualquer alteração quanto aos itens alimentares desde sua instituição. Desde 1959, o Departamento Intersindical de Estatística e Estudos Econômicos (Dieese) realiza uma pesquisa mensal de preços dos itens que compõem a cesta básica de alimentos, sendo utilizada para cálculo do Índice de Custo de Vida (ICV) e estimativa do salário mínimo necessário para atendimento das necessidades básicas do trabalhador e sua família ${ }^{5}$.

Considerando-se que a cesta básica de alimentos ainda é utilizada como padrão de refe- rência para cálculos relacionados ao padrão mínimo de vida e requerimentos de alimentação dos trabalhadores e suas famílias, torna-se necessário investigar os indicadores de viabilidade de acesso, disponibilidade e adequação nutricional dos alimentos que compõem a base das provisões da população trabalhadora do país, especialmente no que tange aos parâmetros de recomendações nutricionais atualmente vigentes. Assim, o objetivo do presente trabalho foi avaliar a evolução dos valores reais cesta básica de alimentos atrelada ao poder aquisitivo do salário mínimo, além de sua disponibilidade nos domicílios brasileiros. Adicionalmente, foram realizadas as análises de composição nutricional e qualidade da dieta baseada no consumo dos itens da cesta básica de alimentos brasileira.

\section{Metodologia}

\section{Desenho do estudo}

O presente trabalho foi realizado a partir da análise quantitativa de natureza exploratória descritiva utilizando documentos oficiais e base de dados secundários de acesso público, empregando-se três abordagens distintas: 1) estudo longitudinal retrospectivo, visando investigar a trajetória da evolução do salário mínimo em termos de poder aquisitivo em relação à cesta básica de alimentos no município de São Paulo; 2) estudo transversal retrospectivo destinado para obtenção de características antropométricas para estimativa das necessidades energéticas da população adulta economicamente ativa; além da investigação da disponibilidade dos itens que compõem a cesta básica de alimentos, considerando a amostra geral de domicílios brasileiros e categorias de quintis de renda per capita; 3) análise documental referente à definição das provisões mínimas estabelecidas para a cesta básica de alimentos brasileira, sendo tais informações destinadas para avaliar a adequação nutricional de dietas baseadas no consumo deste conjunto alimentar.

\section{Análise da evolução dos valores reais do salário mínimo e da cesta básica de alimentos no município de São Paulo}

Foi organizado um banco de dados históricos quanto aos valores nominais e reais do salário mínimo regional para o município de São Paulo, contemplando período de janeiro de 1959 até 
abril de 1984, a partir de bases de dados públicos de instituições de pesquisa ligadas à temática, como Banco Central (Bacen), Instituto Brasileiro de Geografia e Estatística (IBGE) e Instituto de Pesquisa Econômica Aplicada (Ipea), assim como legislação pertinente. Após abril de 1984, foram considerados unificados valores nominais para salário mínimo nacional, conforme Decreto ${ }^{\circ}$ 89.589, de 26 de abril de 1984 .

Simultaneamente, foi feito o levantamento do valor nominal de custeio da cesta básica de alimentos para o município de São Paulo, a partir de dados do Dieese, que constituía base para cálculo do valor do salário mínimo, pautada em pesquisas de consumo alimentar realizadas na década de 1930. A seleção dos parâmetros de análise baseou-se no município de São Paulo como referência em decorrência de disponibilidade de base de dados com período de maior abrangência para valores nominais da cesta básica de alimentos (desde janeiro de 1959). Destaca-se ausência de pesquisa de preços da cesta básica de alimentos pelo Dieese nos meses de março a dezembro de 1964.

Os valores nominais foram deflacionados a partir de metodologia utilizada pelo Ipea, via correção monetária para comparação ao salário mínimo vigente a cada mês, aplicando-se o deflator baseado na composição dos seguintes índices de preços: Índice de Preços ao Consumidor no município de São Paulo, da Fundação Instituto Pesquisas Econômicas da Universidade de São Paulo, IPC-SP/FIPE (entre julho de 1940 e janeiro de 1944); Índice de Preços ao Consumidor no município do Rio de Janeiro da Fundação Getúlio Vargas, IPC-RJ/FGV (entre janeiro de $1944 \mathrm{e}$ janeiro de 1948); Índice Geral de Preços ao Consumidor do Ministério do Trabalho, IGPC/MTb (entre janeiro de 1948 e março de 1979); Índice Nacional de Preços ao Consumidor (INPC) do IBGE até março de 1979; e Índice Nacional de Preços ao Consumidor (INPC) do IBGE a partir de março de 1979 até fevereiro de 2018.

As conversões monetárias decorrentes de alteração da moeda corrente no país foram baseadas em documentação oficial do Banco Central do Brasil, adotando-se a unidade monetária mais recente (Real-R\$) como padrão para mensuração dos valores do salário mínimo e cesta básica de alimentos.

\section{Análise da disponibilidade domiciliar de itens que compõem a cesta básica de alimentos}

Os microdados das Pesquisas de Orçamentos Familiares (POF), realizadas pelo IBGE no período entre 2008 e 2009, foram utilizados para estimativa da disponibilidade per capita de itens da cesta básica de alimentos nos domicílios. A POF tem como objetivo realizar levantamento de informações a respeito de orçamentos domésticos e condições de vida da população, fornecendo inclusive dados sobre distribuição de rendimentos. As análises consideraram o total de cada alimento adquirido divididos pelo total de moradores no domicílio, considerando o total da amostra assim como a categorização dos domicílios por quintis de renda per capita.

\section{Avaliação da composição e adequação nutricional da cesta básica de alimentos}

A partir levantamento de dados disponibilizados pelo Dieese, foi possível obter a composição original proposta da ração-tipo essencial em quantidades mensais de cada um dos alimentos que compõem as cestas básicas regionais e a cesta básica nacional. Em seguida, foram estipuladas provisões mínimas individuais diárias para cada item das cestas básicas.

A composição nutricional de cada cesta básica foi determinada a partir da utilização das seguintes tabelas nacionais de composição nutricional dos alimentos: 1) Tabela Brasileira de Composição de Alimentos, elaborada pelo Núcleo de Estudos e Pesquisas em Alimentação (Nepa) da Universidade Estadual de Campinas; 2) Tabela Brasileira de Composição de Alimentos, elaborada pelo Núcleo de Apoio à Pesquisa em Alimentos e Nutrição (Napan) - Departamento de Alimentos e Nutrição da Faculdade de Ciências Farmacêuticas da USP8

A população de referência para comparação entre requerimentos nutricionais e oferta de nutrientes pela cesta básica foi baseada em indivíduos na faixa etária de 18 a 59 anos. Dados sobre estatura da população de referência foram obtidos a partir dos microdados da Pesquisa de Orçamentos Familiares, conduzida pelo Instituto Brasileiro de Geografia e Estatística em 2008$2009^{6}$, utilizando-se valor de média ponderada do estatura por faixa etária e sexo. A partir da média das estaturas foram calculados o peso de ideal para cada uma das categorias analisadas, utilizando o índice de massa corporal com valo- 


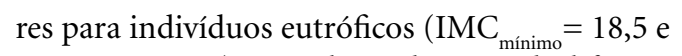
$\left.\mathrm{IMC}_{\text {máximo }}=24,9\right)$. Os valores de peso ideal foram calculados a aplicando-se a equação: Peso ideal $=$ IMC x (estatura média da amostra) ${ }^{2}$.

As estimativas das necessidades energéticas diárias (NED) dos indivíduos da amostra foram elaboradas a partir do cálculo das taxas metabólicas basais (TMB), multiplicado pelo coeficiente de atividade física (Caf). As estimativas das NED foram calculadas utilizando equações para estimação do gasto energético basal preconizado pela Food Agriculture Organization of the United Nations (FAO) ${ }^{9}$.

Para as mulheres na faixa etária entre $18 \mathrm{e}$ 29 anos, o cálculo das necessidades energéticas utilizou a seguinte equação: NED $=\mathrm{C} a f[(14,818$ $x$ Peso médio da amostra) $+486,6]$. Com relação às mulheres com idade entre 30 e 59 anos, a NED foi obtida utilizando a equação: NED = Caf $[(8,126 \times$ Peso médio da amostra $)+845]$. Para a estimativa da NED para os homens com idade entre 18 e 29 anos, utilizou-se a equação: NED = Caf [(15,057 x Peso médio da amostra $)+692,2]$. Entre os homens com idade entre 30 e 59 anos foi utilizada a seguinte equação para estimativa da NED: NED = Caf [(11,472 x Peso médio amostra) $+873,1]$. Para todas as estimativas das NED foram atribuídos o Caf para indivíduos sedentários, com valor igual a 1,4 .

Os valores de energia, macronutrientes e micronutrientes das dietas compostas pelos itens alimentares das cestas básicas foram analisados em comparação com recomendações nutricionais atualmente vigentes, de acordo com Dietary Reference Intakes (DRI) ${ }^{10}$.

\section{Avaliação da qualidade global da dieta}

Os atributos qualitativos da "ração-tipo" foram avaliados pela metodologia do Índice de Qualidade da Dieta Revisado (IQD-R) adaptado à população brasileira ${ }^{11}$, a partir da análise de componentes da composição alimentar e nutricional (Quadro 1).

O IQD-R permite avaliar potenciais benefícios à saúde provenientes dos diferentes perfis de consumo alimentar em estudo. O cálculo do IQD -R apresenta escala de 0 (pior qualidade da dieta) a 100 (melhor qualidade da dieta).

As provisões mensais de cada item da cesta básica de alimentos foram convertidas em quantidades relativas ao consumo individual diário para estimativa do valor energético e teor de sódio, gordura saturada e açúcar, componentes necessários para mensuração do IQD-R.
O cálculo do escore IQD-R em cada componente avaliado foi realizado seguindo roteiro de codificação de alimentos e ingredientes elaborado pelo Grupo de Pesquisa de Avaliação do Consumo Alimentar da Faculdade de Saúde Pública (GAC-FSPUSP).

\section{Organização dos bancos de dados, análises estatísticas e cálculos dos parâmetros de avaliação nutricional}

As compilações dos bancos de microdados da POF 2008-2009 foram efetuadas no programa Stata ${ }^{\circledR}$, versão 13.0, a partir do módulo survey, no qual foi aplicado o fator de expansão da amostra para obtenção da representatividade de pessoas e domicílios.

A análise de variância de uma via (One-Way ANOVA), complementada com teste de Bonferroni, foi utilizada para comparação de médias entre os grupos, sendo tais testes utilizados para determinar possíveis diferenças dos valores de médios obtidos de cada variável analisada (número de pessoas residentes no domicílio, renda per capita e disponibilidade semanal per capita de cada item da cesta básica de alimentos) entre os quintis de domicílio classificados pela renda per capita. Adotou-se o nível de significância para análises estatísticas referentes ao teste de hipótese nula igual ou menor a $5 \%(\mathrm{p}<0,05)$.

Todos cálculos para estimativa das necessidades energéticas dos indivíduos da amostra, medidas de composição nutricional e aplicação do IQD-R para avaliação as dietas baseadas na cesta básica de alimentos também foram realizadas no programa Stata ${ }^{\circledR}$, versão 13.0.

\section{Resultados}

A partir da análise da evolução dos valores reais do salário mínimo e da cesta básica de alimentos no município de São Paulo, foi possível avaliar a evolução do poder aquisitivo deste padrão de remuneração mínimo vigente, em relação à capacidade aquisição dos itens da ração essencial mínima (Figura 1). Em janeiro de 1959, o valor do salário mínimo encontrava-se em uma fase de ganhos reais, entretanto, no período entre $1960 \mathrm{e}$ 1964 ocorreram perdas importantes do valor real do salário mínimo. Entre 1965 e 1975, os valores reais do salário mínimo apresentaram menores flutuações. O período crítico que culminou na diminuição dos valores reais do salário mínimo ocorreu entre 1985 e 1991. Em agosto de 1991, o 
Quadro 1. Componentes para avaliação do IQD-R da cesta básica de alimentos.

\begin{tabular}{|c|c|c|c|c|}
\hline \multirow[b]{2}{*}{ Componente } & \multirow{2}{*}{$\begin{array}{c}\text { Critério para } \\
\text { pontuação mínima }\end{array}$} & \multirow{2}{*}{$\begin{array}{l}\text { Critério para } \\
\text { pontuação } \\
\text { intermediária }\end{array}$} & \multicolumn{2}{|c|}{ Critério para pontuação máxima } \\
\hline & & & Porções & Pontos \\
\hline Frutas totais & \multirow{8}{*}{$\begin{array}{l}\text { Ausência de } \\
\text { consumo } \\
0 \text { ponto }\end{array}$} & \multirow[t]{8}{*}{ Cálculo proporcional } & 1,0 porção/1.000 kcal & \multirow[t]{4}{*}{5} \\
\hline Frutas integrais & & & 0,5 porção/1.000 kcal & \\
\hline Vegetais totais & & & 1,0 porção/1.000 kcal & \\
\hline $\begin{array}{l}\text { Vegetais verde escuros, } \\
\text { alaranjados e legumes }\end{array}$ & & & 0,5 porção/1.000 kcal & \\
\hline Cereais totais & & & 3,0 porções/1.000 kcal & \multirow[t]{6}{*}{10} \\
\hline Carnes, ovos e leguminosas & & & 1,5 porção/1.000 kcal & \\
\hline Leite e derivados & & & 1,0 porção/1.000 kcal & \\
\hline Óleos & & & 0,5 porção/1.000 kcal & \\
\hline Gordura saturada & $\begin{array}{l}\geq 15 \% \text { do VET } \\
0 \text { pontos }\end{array}$ & $\begin{array}{l}10 \% \text { do VET } \\
8 \text { pontos }\end{array}$ & $\leq 7 \%$ do VET & \\
\hline Sódio & $\begin{array}{l}\geq 2,0 \mathrm{~g} / 1.000 \mathrm{kcal} \\
0 \text { pontos }\end{array}$ & $\begin{array}{l}1 \mathrm{~g} / 1.000 \mathrm{kcal} \\
8 \text { pontos }\end{array}$ & $\leq 0,75 \mathrm{~g} / 1.000 \mathrm{kcal}$ & \\
\hline Gord A $\mid \mathrm{A}$ & $\begin{array}{l}\geq 35 \% \text { do VET } \\
0 \text { pontos }\end{array}$ & Cálculo proporcional & $\leq 10 \%$ do VET & 20 \\
\hline
\end{tabular}

Legenda: VET = Valor Energético Total; Gord A|A = Energia provenientes de gordura sólida, álcool e açúcar de adição. Fonte: Previdelli et al. ${ }^{11} \mathrm{e}$ Wendpap et al. ${ }^{12}$.

salário mínimo estimado era de $\mathrm{R} \$ 273,68$, sendo este o menor valor real estimado considerando o período analisado. Em fevereiro de 2018 (período de referência para a análise), o salário mínimo corresponde a $\mathrm{R} \$ 954,00$.

$\mathrm{Na}$ análise da evolução dos valores reais da cesta básica no município de São Paulo, foi verificado que períodos de alta de preços para aquisição dos itens da ração mínima, especialmente entre o período de 1973 a 1995 (Figura 1). Entre 1983 a 1995 foi o período de maior dificuldade para a aquisição dos itens da cesta básica de alimentos, de modo que a o valor para a aquisição de tais itens chegava a aproximadamente 1,4 vezes o valor do salário mínimo em abril de 1995.

A partir da análise dos microdados da POF 2008-2009 sobre a aquisição de produtos efetuados pelos domicílios brasileiros, foi verificado maior número de domicílios que adquiriram pão francês, carne bovina, leite, tomate, café em pó e arroz (Quadro 2). Por sua, os alimentos adquiridos em menor frequência pelos domicílios foram manteiga, banana, farinha de trigo, batata, feijão e açúcar.

Foi possível observar que os domicílios nos menores quintis de renda per capita (Q1 e Q2) apresentam maior número de pessoas residentes, se comparado aos domicílios nas categorias de alta renda (Q4 e Q5) (Quadro 2). Em contrapartida, foi verificado que os domicílios de baixa renda possuem menor disponibilidade de todos os itens da cesta básica de alimentos, em relação aos domicílios mais abastados (Quadro 2).

$\mathrm{O}$ valor energético proveniente dos itens da cesta básica de alimentos nacional resulta em aproximadamente $3.044 \mathrm{kcal}$ por dia. A proporção energética de carboidratos (61\%) e lipídios (26\%) situa-se nas faixas recomendadas para consumo de macronutrientes, havendo a constatação de inadequação para a ingestão de proteínas (13\%) (Quadro 3).

A partir da estimativa das necessidades energéticas de um trabalhador médio brasileiro com idade entre 18 e 29 anos, haveria necessidade da ingestão de 1.580 a $1.882 \mathrm{kcal}$ por dia entre mulheres; enquanto que entre homens, as necessidades energéticas estimadas variam entre 1.703 a $2.392 \mathrm{kcal}$ por dia (Quadro 3). Considerando os indivíduos na faixa etária entre 30 e 59 anos, o consumo energético recomendado para as mulheres encontra na faixa 1.703 a $1.882 \mathrm{kcal}$ diários; já para os homens, estima-se a ingestão diária ideal entre 2.091 a $2.392 \mathrm{kcal}$.

Assim, considerando hipótese do consumo diário dos itens componentes da cesta básica de alimentos nas proporções estabelecidas, haveria excesso de consumo energético em relação às necessidades estimadas para população.

Por outro lado, o padrão de dieta estipulado pelos componentes da cesta básica de alimentos resultaria em ingestão insuficiente de cálcio, potássio e vitamina A (em equivalente de atividade 

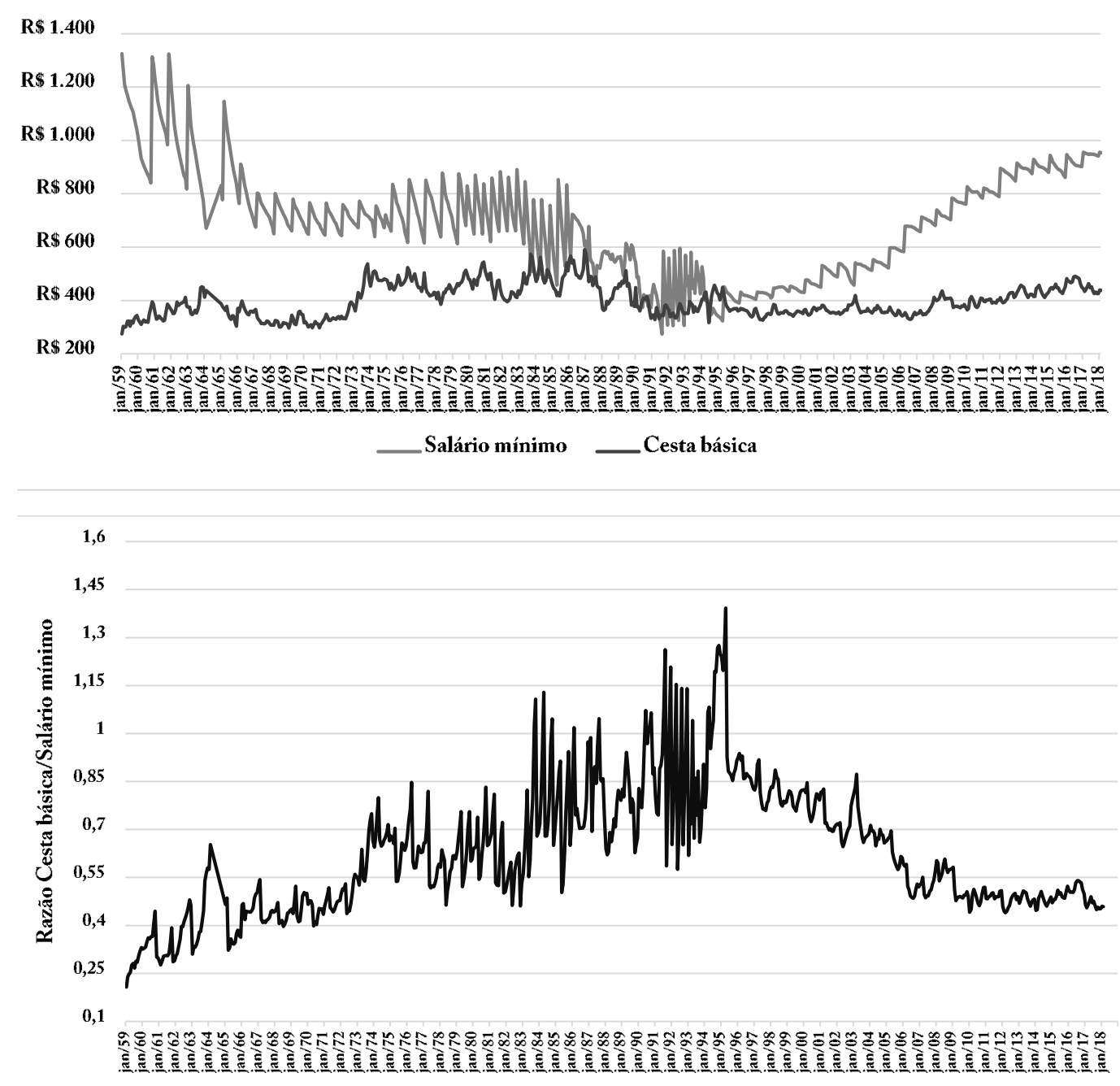

Figura 1. Evolução dos valores do salário mínimo e da cesta básica de alimentos no município de São Paulo. 1959-2018.

Fonte: Elaboração própria, a partir da série temporal do salário mínimo real, do acervo de índices de inflação (IPEA) e da Pesquisa Nacional da Cesta Básica de Alimentos (DIEESE).

de retinol - RAE); assim como consumo excessivo de sódio. Entre mulheres em idade fértil, também ocorreria ingestão de ferro inferior às recomendações atuais (Quadro 4).

A avaliação da qualidade da dieta apontou diferenças entre cestas de alimentos regionais, a partir das pontuações do IQD-R (Quadro 4). A região 2 apresentou maior escore de qualidade da alimentação (52,03), enquanto a cesta básica de alimentos nacional apresentou menor pontuação $(49,11)$.
O excesso de açúcar de adição, a ausência de cereais integrais e o baixo consumo de vegetais, leite e derivados foram principais fatores contribuintes à menor pontuação das cestas de básicas de alimentos no escore geral de qualidade da dieta.

A maior quantidade de alimentos de elevado teor energético com alta proporção de gordura, tais como óleo e manteiga, resultou na baixa pontuação da cesta básica de alimentos nacional, contribuindo para maior aporte energético total, ocorrendo assim menor participação proporcional dos demais grupos alimentares na dieta. 


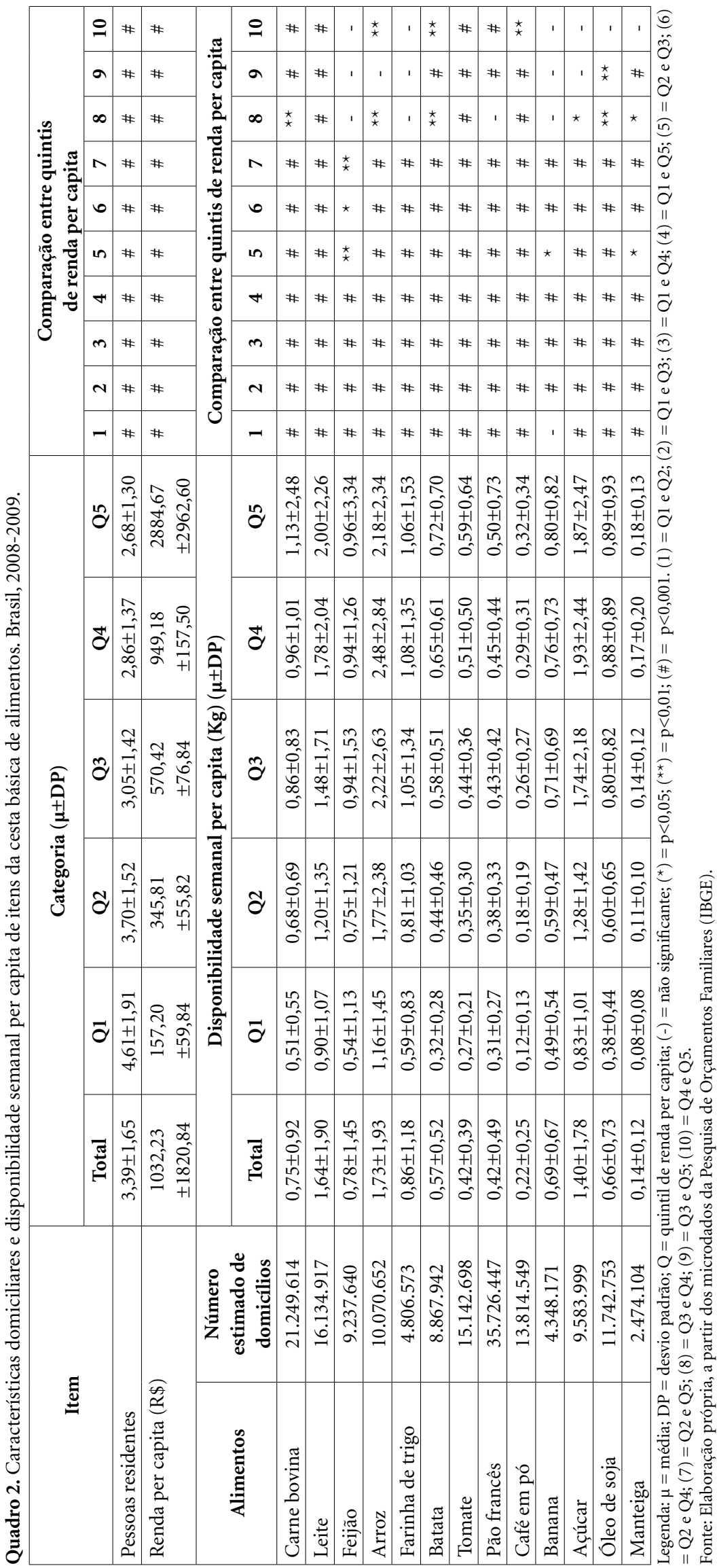


Quadro 3. Estimativas das necessidades energéticas individuais em comparação com os valores energéticos obtidos para o consumo de itens da cesta básica de alimentos.

\begin{tabular}{|c|c|c|c|c|c|c|c|c|}
\hline \multirow{3}{*}{ Categoria } & \multicolumn{4}{|c|}{$\begin{array}{c}\text { Características antropométricas e } \\
\text { recomendações nutricionais }{ }^{\star}\end{array}$} & \multicolumn{4}{|c|}{$\begin{array}{c}\begin{array}{c}\text { Cestas básicas de alimentos } \\
\text { (dispêndio diário) }\end{array} \\
\end{array}$} \\
\hline & \multicolumn{2}{|c|}{18 a 29 anos } & \multicolumn{2}{|c|}{30 a 59 anos } & \multirow{2}{*}{ Nacional } & \multirow{2}{*}{\begin{tabular}{|c|} 
Região \\
1
\end{tabular}} & \multirow{2}{*}{$\begin{array}{l}\text { Região } \\
2\end{array}$} & \multirow{2}{*}{\begin{tabular}{|c|} 
Região \\
3
\end{tabular}} \\
\hline & Mulheres & Homens & Mulheres & Homens & & & & \\
\hline População estimada & 19.759 .454 & 19.697 .588 & 37.873 .654 & 33.927 .148 & & & & \\
\hline Altura $(\mathrm{m})(\mu \pm \mathrm{DP})$ & $1,53 \pm 0,36$ & $1,73 \pm 0,24$ & $1,57 \pm 0,19$ & $1,71 \pm 0,07$ & & & & \\
\hline Peso ideal estimado (kg) & $43,3-58,3$ & $55,4-74,5$ & $45,6-61,4$ & $54,1-72,8$ & & & & \\
\hline Energia $(\mathrm{kcal})$ & $1.580-1.890$ & $2.136-2.540$ & $1.703-1.882$ & 2.091-2.392 & 3.044 & 2.875 & 2.865 & 2.946 \\
\hline Proteínas (\%VET) & & & & 25-35 & 13 & 13 & 12 & 13 \\
\hline Lipídios (\%VET) & & & & $15-30$ & 26 & 24 & 23 & 26 \\
\hline Carboidratos (\%VET) & & & & $45-65$ & 61 & 63 & 65 & 61 \\
\hline
\end{tabular}

${ }^{*}$ Referem-se às necessidades energéticas estimadas utilizando equação de metabolismo basal da FAO e recomendações de macronutrientes das DRI. $\mu$ = média; DP = desvio padrão; VET = valor energético total. Região 1 = Estados do Espírito Santo, Minas Gerais, Rio de Janeiro, São Paulo, Goiás e Distrito Federal. Região 2 = Estados de Alagoas, Bahia, Ceará, Maranhão, Paraíba, Pernambuco, Piauí, Rio Grande do Norte, Sergipe, Acre, Amapá, Amazonas, Pará, Rondônia, Roraima e Tocantins. Região $3=$ Estados do Paraná, Rio Grande do Sul, Santa Catarina, Mato Grosso e Mato Grosso do Sul. Nacional = Cesta normal média para massa trabalhadora em atividades diversas em território nacional.

Fonte: Elaboração própria, a partir de Dietary Reference Intakes, Pesquisa Nacional da Cesta Básica de Alimentos (DIEESE), TACO (UNICAMP) e TBCA (USP).

\section{Discussão}

Ao longo de suas trajetórias, o salário mínimo e a cesta básica de alimentos sofreram transformações em suas aplicabilidades e efetividades como políticas públicas, havendo constantes perdas de suas relevâncias no contexto alimentar devido aos eventos políticos, econômicos, assim como nas mudanças no padrão alimentar da população ocorridos ao longo da história recente do país.

Os resultados corroboram com a constatação da deterioração da relevância do salário mínimo para assegurar as necessidades básicas com alimentação, em decorrência da ausência atualizações monetárias em decorrência da corrosão do poder aquisitivo pela alta da inflação e fracasso dos choques heterodoxos realizados na forma de planos econômicos inconsistentes ${ }^{13}$.

Os dados apresentados a respeito do poder aquisitivo do salário mínimo em relação à capacidade de aquisição da cesta básica de alimentos no município de São Paulo demonstram a existência de períodos de incapacidade de custeio para as despesas básicas com alimentação, sendo estas preconizadas por lei como necessidades vitais dos trabalhadores e suas famílias. Existe ainda o fato de que a partir da Constituição Federal de 1946, o salário mínimo passou a ser o padrão de remuneração mínima para os trabalhadores e suas famílias, mas sem este padrão de remuneração-base sofrer qualquer adequação quanto os valores que viabilizem o atendimento das provisões mínimas para suprir as necessidades básicas das famílias ${ }^{14}$.
A insegurança alimentar é potencialmente expressa pela dificuldade de acesso aos alimentos, estando associada ao baixo nível de renda e baixa escolaridade ${ }^{15}$. Os resultados mostrados no presente estudo constatam períodos em que o salário mínimo não é capaz de custear a compra de itens que compõem a alimentação. Deste modo, a defasagem do salário mínimo frente a sua desvinculação dos custos para aquisição de itens da cesta básica de alimentos poderia desencadear situações de insegurança alimentar, especialmente nos domićlios cuja a renda per capita seja equivalente ou inferior a este padrão de rendimentos.

A incapacidade do salário mínimo de custear as necessidades básicas com alimentação viola o direito humano à alimentação adequada (DHAA), sendo este o direito inerente a todas as pessoas de ter acesso físico, econômico, regular, permanente e irrestrito, à alimentação adequada ou aos meios para obter alimentos seguros e saudáveis em quantidade e qualidade adequadas, sem comprometer outros direitos fundamentais ${ }^{16}$.

Pelo fato de os inquéritos realizados pelas POFs investigarem a aquisição semanal dos produtos realizada pelos domicílios, o comportamento de aquisição para alguns itens pode explicar a frequência dos registros de alguns alimentos pelos domicílios. No caso das estimativas de disponibilidade per capita domiciliar para o pão francês, acaba sendo comum a aquisição diária deste item. Enquanto que no caso de itens com menor perecibilidade, tais como o arroz e o fei- 
Quadro 4. Valores totais das provisões mínimas individuais, macronutrientes, micronutrientes e pontuações do IQD-R referentes ao consumo individual diário de itens das cestas básicas de alimentos.

\begin{tabular}{|c|c|c|c|c|}
\hline \multirow{2}{*}{ Item } & \multicolumn{4}{|c|}{ Categoria } \\
\hline & Nacional & Região 1 & Região 2 & Região 3 \\
\hline \multicolumn{5}{|l|}{ Provisões diárias estimadas } \\
\hline Carne bovina $(\mathrm{g})$ & 200 & 200 & 150 & 220 \\
\hline Leite $(\mathrm{ml})$ & 500 & 250 & 200 & 250 \\
\hline Feijão (g) & 150 & 150 & 150 & 150 \\
\hline Arroz (g) & 100 & 100 & 120 & 100 \\
\hline Farinha de trigo $(\mathrm{g})$ & 50 & 50 & 100 & 50 \\
\hline Batata $(\mathrm{g})$ & 200 & 200 & - & 200 \\
\hline Tomate $(\mathrm{g})$ & 300 & 300 & 400 & 300 \\
\hline Pão francês $(\mathrm{g})$ & 200 & 200 & 200 & 200 \\
\hline Café em pó (g) & 20 & 20 & 10 & 20 \\
\hline Banana $(\mathrm{g})$ & 120 & 120 & 120 & 120 \\
\hline Açúcar (g) & 100 & 100 & 100 & 100 \\
\hline Óleo de soja (g) & 25 & 25 & 25 & 30 \\
\hline Manteiga (g) & 25 & 25 & 25 & 25 \\
\hline \multicolumn{5}{|l|}{ Nutrientes } \\
\hline Proteínas (g) & 102,09 & 93,89 & 84,95 & 97,77 \\
\hline Lipídios (g) & 87,51 & 78,01 & 72,87 & 84,2 \\
\hline Colesterol (mg) & 218,47 & 192,24 & 158,03 & 203,83 \\
\hline Carboidratos (g) & 462 & 449,35 & 467,31 & 449,35 \\
\hline Fibras $(\mathrm{g})$ & 38,74 & 38,74 & 33,96 & 38,74 \\
\hline Cálcio (mg) & $771,18^{\star}$ & $464,73^{\star}$ & $401,10^{*}$ & $465,25^{\star}$ \\
\hline Magnésio (mg) & 363,5 & 339,41 & 313,97 & 342,24 \\
\hline Manganês (mg) & 4,03 & 4,03 & 4,08 & 4,03 \\
\hline Fósforo (mg) & $1.411,56$ & $1.206,62$ & $1.091,21$ & $1.238,14$ \\
\hline Ferro (mg) & $12,33^{*}$ & $12,33^{*}$ & $10,76^{*}$ & $12,68^{*}$ \\
\hline Sódio (mg) & $1.863,36^{\star *}$ & $1.703,96^{\star \star}$ & $1.649,25^{\star \star}$ & $1.713,68^{\star \star}$ \\
\hline Potássio (mg) & $3.973,51^{\star}$ & $3.640,54^{*}$ & $3.000,90^{*}$ & $3.688,00^{*}$ \\
\hline Cobre (mg) & 1,61 & 1,56 & 1,32 & 1,57 \\
\hline Zinco (mg) & 19,95 & 19 & 15,91 & 20,27 \\
\hline Vitamina A - RAE (mcg) & $105,09^{*}$ & $105,09^{*}$ & $130,93^{*}$ & $105,55^{\star}$ \\
\hline Tiamina $(\mathrm{mg})$ & 2,21 & 2,11 & 2,12 & 2,14 \\
\hline Riboflavina (mg) & 2,99 & 2,39 & 2,16 & 2,43 \\
\hline Piridoxina (mg) & 1,82 & 1,82 & 1,54 & 1,83 \\
\hline Niacina $(\mathrm{mg})$ & 24,87 & 21,07 & 17,63 & 21,94 \\
\hline Vitamina C (mg) & 151,71 & 151,71 & 110,76 & 151,71 \\
\hline Lip. Sat. (mg) & 31,81 & 28,31 & 27,63 & 29,61 \\
\hline Monoinsat. (mg) & 21,5 & 19,75 & 19,42 & 21,39 \\
\hline Polinsat. (mg) & 18,93 & 18,68 & 18,65 & 21,7 \\
\hline Ácido linoleico (g) & 16,81 & 16,71 & 16,71 & 19,43 \\
\hline Ácido alfa-linoleico (g) & 1,93 & 1,88 & 1,87 & 2,17 \\
\hline Pontuação total do IQD-R & 49,11 & 49,6 & 52,03 & 49,18 \\
\hline \multicolumn{5}{|c|}{ Adequação dos componentes do IQD-R (\%) } \\
\hline Frutas totais & 38,3 & 40,11 & 46,8 & 38,97 \\
\hline Frutas integrais & 76,59 & 80,22 & 93,59 & 77,95 \\
\hline Vegetais totais & 36,14 & 37,86 & 44,17 & 36,78 \\
\hline Vegetais verdes e alaranjados & 0 & 0 & 0 & 0 \\
\hline Cereais totais & 50 & 50 & 50 & 50 \\
\hline Carnes & 53,8 & 56,35 & 65,75 & 54,76 \\
\hline Leite e derivados & 10,04 & 10,52 & 12,27 & 10,22 \\
\hline
\end{tabular}


Quadro 4. Valores totais das provisões mínimas individuais, macronutrientes, micronutrientes e pontuações do IQD-R referentes ao consumo individual diário de itens das cestas básicas de alimentos.

\begin{tabular}{|l|c|c|c|c|}
\hline \multirow{2}{*}{\multicolumn{1}{|c|}{ Item }} & \multicolumn{4}{c|}{ Categoria } \\
\cline { 2 - 5 } & Nacional & Região 1 & Região 2 & Região 3 \\
\hline Óleos & 100 & 100 & 100 & 100 \\
\hline Gordura saturada & 100 & 100 & 100 & 100 \\
\hline Sódio & 100 & 100 & 100 & 100 \\
\hline Gord A|A & 1,78 & 0 & 0 & 0 \\
\hline
\end{tabular}

RAE $=$ Equivalentes de atividade de retinol; Lip.Sat. = lipídios saturados; Monoinsat. $=$ lipídios monoinsaturados; Polinsat. $=$ lipídios poli-insaturados. ${ }^{\star}$ Micronutriente em quantidade insuficiente; ${ }^{\star *}$ Micronutriente em excesso de quantidade.

Fonte: Elaboração própria, a partir de: i) Decreto Lei no 399 de 1938; ii) Pesquisa Nacional da Cesta Básica de Alimentos (DIEESE), iii) TACO (UNICAMP), TBCA (USP) e; iv) metodologia do cálculo para IQD-R (Previdelli et al. ${ }^{11}$ ).

jão, ocorreu menor registro de aquisição no âmbito domiciliar no inquérito realizado pelo IBGE, possivelmente pelo fato destes alimentos serem estocados com maior facilidade.

A análise da qualidade nutricional da cesta básica buscou demonstrar a necessidade de revisão da composição da alimentação voltada para suprir necessidades básicas da população. O estudo realizado por Barretto et al. ${ }^{17}$ possui grande relevância da temática da pesquisa, ao se investigar a composição nutricional da cesta básica idealizada por Dieese e Procon, a qual continha 22 itens alimentares. Dentre os principais achados dessa pesquisa, destacam-se as insuficiências para os minerais cálcio, magnésio, ferro, zinco e iodo, e ainda para as vitaminas A, C, B2 e B6 ${ }^{17}$. Este trabalho também efetuou o cálculo de complementação alimentar a partir da inclusão de outros alimentos pesquisados em inquéritos realizados para a elaboração do Índice de Preços ao consumidos (IPC) realizado pela Fundação Instituto de Pesquisas Econômicas (Fipe), e também pela POF realizada no município de São Paulo na década de 1990. No trabalho publicado por Passos et al. ${ }^{18}$ também foi analisada a adequação nutricional da cesta básica de alimentos nacional, constatando valores do teor energético total e lipídeos acima das recomendações, estando cálcio, vitamina A abaixo do recomendado, estando a vitamina $\mathrm{C}$ inadequadas para os homens ${ }^{18}$.

É importante destacar que o presente estudo difere dos trabalhos publicados anteriormente por tratar-se da primeira investigação a utilizar integralmente tabelas brasileiras de composição nutricional de alimentos, cujos valores apresentam maior fidedignidade aos alimentos consumidos no país, possibilitando mensuração de dados de consumo nutricional com maior confiabilidade. Um outro ponto importante realizado neste estudo foi a análise da adequação nutricional do consumo alimentar com base no consumo dos itens da cesta básica nacional, e também para as provisões determinadas para três regiões do país, segundo o Decreto-Lei 399 de 30 de abril de 1938. Estas provisões ainda seguem como padrões de referência para pesquisas de aferição de preços e custo de vida realizadas por entidades tais como Dieese. Salienta-se ainda a aplicação de uma segunda abordagem de avaliação nutricional, baseada em critérios qualitativos utilizando o IQD-R. A aplicação desse índice traz consigo a capacidade de avaliar de forma sintética a adequação do consumo de diferentes grupos de alimentos, de acordo com a densidade energética ofertada na dieta.

Considerando a concepção inicial da cesta básica, a qual foi elaborada para o atendimento das necessidades básicas de alimentação de um trabalhador, verificou-se que as dietas baseadas no consumo dos itens listados na ração-tipo apresentariam elevado teor energético, comparado às estimativas das necessidades energéticas para homens e mulheres adultos que compõem a maior parte população economicamente ativa. Além do mais, tais dietas apresentam teor reduzido de cálcio, potássio e vitamina A (mensurada em RAE). A inadequação no consumo de tais nutrientes também foi identificada no consumo alimentar pessoal, a partir de inquérito realizado no contexto da POF 2008-200919.

Observou-se também que uma dieta baseada no consumo das provisões mínimas diárias dos itens da cesta básica de alimentos resultaria em excesso de ingestão de sódio, similarmente ao padrão alimentar brasileiro atual, que contém aproximadamente o dobro de sódio recomendado no contexto das $\mathrm{DRI}^{20}$.

Os resultados apresentados também apontam para a carência de ferro na dieta baseada em itens da cesta básica, em comparação às re- 
comendações nutricionais atuais para mulheres em idade fértil. No contexto da saúde pública, verifica-se relevante papel da ingestão de ferro na prevenção da anemia ferropriva e ferropênica entre mulheres em idade reprodutiva e gestantes, grupos vulneráveis à ocorrência da deficiência nutricional $^{21,22}$.

Ademais, haveria necessidade de revisar a composição da cesta básica para inclusão de maior variedade de grupos de alimentos, sendo notório déficit de alimentos in natura, como verduras, frutas totais, cereais totais, cereais integrais, leite e derivados.

Considerando conjunto dos resultados, as quantidades de alimentos que compõem cestas básicas de alimentos no Brasil deveriam ser revisadas para contemplar as necessidades dos grupos familiares, de maneira proporcional ao número e composição demográfica dos integrantes no domicílio.

A cesta básica de alimentos apresenta similaridade com bases da alimentação atual da população brasileira, especialmente considerando as altas proporções de energia disponíveis nos itens que compõem a ração-tipo essencial ${ }^{23,24}$.

A alta prevalência no consumo de carnes, leite e feijão e a baixa prevalência do consumo de frutas e hortaliças são características importantes que sinalizam qualidade da alimentação de indivíduos adultos no Brasil ${ }^{25,26}$.

Nas últimas décadas, mudanças importantes vêm ocorrendo no padrão alimentar brasileiro, especialmente em termos do aumento da disponibilidade fontes proteicas de alto valor biológico, gordura animal e alimentos processados, assim como significativo teor de açúcares livres e lipídios totais ${ }^{24}$.

Tais alterações no padrão alimentar da população brasileira corroboram ocorrência e consolidação da transição nutricional, caracterizada pelo aumento da carga de doenças crônicas não transmissíveis como obesidade, diabetes, anemia e câncer ${ }^{27-29}$.

\section{Conclusões}

O salário mínimo frente à capacidade de aquisição da cesta básica mostra-se como uma medida de renda inviável para o custeio das necessidades básicas com alimentação.

A dieta com base no consumo dos itens da cesta básica de alimentos apresenta excessiva oferta energética, considerando-se os requerimentos nutricionais para indivíduos adultos no
Brasil; entretanto, apresenta baixo conteúdo de cálcio, potássio e vitamina $\mathrm{A}$, além de apresentar quantidade insuficiente de ferro para suprimento das necessidades nutricionais de mulheres em idade fértil.

A composição da cesta básica de alimentos mostra-se deficitária em termos de alimentos in natura ou minimamente processados, especialmente frutas totais, frutas integrais, vegetais totais, vegetais verde escuros, alaranjados e legumes, cereais totais, leite e derivados, além de apresentar excesso de gordura e açúcar de adição.

Diante de tais achados, ressalta-se a importância da revisão da composição das cestas básicas de alimentos regionais e nacional, de forma a cumprir requerimentos nutricionais da população brasileira, buscando promover padrões alimentares saudáveis. $\mathrm{O}$ estabelecimento de ajustes das provisões mínimas destinadas à alimentação bem como o suprimento dos grupos alimentares de origem vegetal in natura e minimamente processados, além de do grupo de leite e derivados, permitirá aprimorar as pesquisas voltadas para o cálculo das estimativas dos gastos familiares quanto ao custo de vida. Por fim, sugere-se que estudos futuros investiguem o papel de políticas de acesso aos demais alimentos in natura na garantia de complementação do conteúdo nutricional da cesta básica.

\section{Colaboradores}

ABC Santana participou ativamente das etapas de concepção, delineamento metodológico, realização das análises, interpretação dos resultados e elaboração do manuscrito final. FM Sarti orientou o estudo e participou das etapas de concepção do estudo, verificação da metodologia, acompanhamento das análises, interpretação e discussão dos resultados e revisão do manuscrito final.

\section{Agradecimentos}

Aperfeiçoamento de Pessoal de Nível Superior (CAPES) pela concessão de bolsa e ao Conselho Nacional de Desenvolvimento Científico e Tecnológico $(\mathrm{CNPq})$ pelo apoio financeiro para realização do presente trabalho. 


\section{Referências}

1. Vasconcelos FAG. Combate à fome no Brasil: uma análise histórica de Vargas a Lula. Rev Nutr 2005; 18(4):439-457.

2. Castro J. Condições de vida das classes operárias do Recife. Rev Arq Municipal 1932; 18:167-78.

3. Stepan NL. A hora da eugenia. Raça, gênero e nação na América Latina. Rio de Janeiro: Fiocruz; 2005.

4. Magalhães R. Fome: uma (re)leitura de Josué de Castro. Rio de Janeiro: Editora Fiocruz; 1997.

5. Departamento Intersindical de Estatística e Estudos Econômicos (DIEESE). Metodologia da Pesquisa Nacional da Cesta Básica de Alimentos. São Paulo: DIEESE; 2016.

6. Brasil. Instituito Brasileiro de Geografia e Estatística (IBGE). Pesquisa de Orçamentos Familiares: Antropometria e Estado Nutricional de Crianças, Adolescentes e Adultos no Brasil. Rio de Janeiro: IBGE; 2010.

7. Núcleo de Estudos e Pesquisas em Alimentação. Tabela brasileira de composição de alimentos. Campinas: UNICAMP; 2011.

8. Núcleo de Apoio à Pesquisa em Alimentos e Nutrição. Tabela brasileira de composição de alimentos. São Paulo: USP; 2018.

9. Schofield W. Predicting basal metabolic rate, new standards and review of previous work. Hum Nutr Clinical Nutr 1985; 39:5-41.

10. Otten JJ, Hellwig JP, Meyers LD. Dietary reference intakes: the essential guide to nutrient requirements. Washington: National Academies Press; 2006.

11. Previdelli ÁN, Andrade SC, Pires MM, Ferreira SRG, Fisberg RM, Marchioni DM. Índice de Qualidade da Dieta Revisado para população brasileira. Rev Saúde Pública 2011; 45(4):794-798.

12. Wendpap LL, Ferreira MG, Melo PR, Pereira RA, Loureiro AS, Gonçalves-Silva RMV. Qualidade da dieta de adolescentes e fatores associados. Cad Saúde Pública 2014; 30(1):97-106.

13. Gremaud AP, Vasconcellos MAS, Toneto R. Economia brasileira contemporânea. São Paulo: Ed. Atlas; 2015.

14. Mattoso J. Mínimo salário mínimo. São Paulo; Revista São Paulo em Perspectiva; 1988.

15. Bezerra TA, Olinda RA, Pedraza DF. Insegurança alimentar no Brasil segundo diferentes cenários sociodemográficos. Cien Saude Colet 2017; 22(2):637-651.

16. Leão M. O Direito Humano à Alimentação Adequada e o Sistema Nacional de Segurança Alimentar e Nutricional. Brasília: ABRANDH; 2013.

17. Barretto SA, Cyrillo DC, Cozzolino SM. Análise nutricional e complementação alimentar de cesta básica derivada do consumo. Rev Saúde Pública 1998; 32(1):29-35.

18. Passos KEd, Bernardi JR, Mendes KG. Análise da composição nutricional da Cesta Básica brasileira. Cien Saude Colet 2014; 19(5):1623-1630.

19. Brasil. Instituto Brasileiro de Geografia e Estatística (IBGE). Pesquisa de Orçamentos Familiares 2008-2009: análise do consumo alimentar pessoal no Brasil. Rio de Janeiro: IBGE; 2011.
20. Sarno F, Claro RM, Levy RB, Bandoni DH, Monteiro CA. Estimativa de consumo de sódio pela população brasileira, 2008-2009. Rev Saúde Pública 2013; 47(3):571-578.

21. Lopes SM, Freitas IR. Anemia ferropriva/ferropênica em gestantes: uma revisão integrativa da literatura. Rev UninCor 2015; 13(1):442-451.

22. Bezerra AGN, Leal VS, Lira PIC, Oliveira JS, Costa EC, Menezes RCE, Campos FACS, Andrade MIS. Anemia e fatores associados em mulheres de idade reprodutiva de um município do Nordeste brasileiro. Rev Bras Epidemiol 2018; 21:e180001.

23. Levy-Costa RB, Sichieri R, Pontes ND, Monteiro CA Household food availability in Brazil: distribution and trends (1974-2003). Rev Saude Publica 2005; 39(4):530-540.

24. Levy RB, Claro RM, Mondini L, Sichieri R, Monteiro CA. Regional and socioeconomic distribution of household food availability in Brazil, in 2008-2009. Rev Saude Publica 2012; 46(1):6-15.

25. Claro RM, Santos MAS, Oliveira TP, Pereira CA, Szwarcwald CL, Malta DC. Consumo de alimentos não saudáveis relacionados a doenças crônicas não transmissíveis no Brasil: Pesquisa Nacional de Saúde, 2013. Epidemiol Serv Saúde 2015; 24(2):257-265.

26. Jaime PC, Stopa SR, Oliveira TP, Vieira ML, Szwarcwald CL, Malta DC. Prevalência e distribuição sociodemográfica de marcadores de alimentação saudável, Pesquisa Nacional de Saúde, Brasil 2013. Epidemiol Serv Saúde 2015; 24(2):267-276.

27. Batista Filho M, Rissin A. A transição nutricional no Brasil: tendências regionais e temporais. Cad Saúde Pública 2003; 19(Supl. 1):S181-S191.

28. Batista Filho M, Souza AI, Miglioli TC, Santos MC. Anemia e obesidade: um paradoxo da transição nutricional brasileira. Cad Saúde Pública 2008; 24(Supl. 2):S247-S257.

29. Schmidt MI, Duncan BB, Silva GAE, Menezes AM, Monteiro CA, Barreto SM, Chor D, Menezes PR. Health in Brazil 4 Chronic non-communicable diseases in Brazil: burden and current challenges. Lancet 2011; 377(9781):1949-19461.

Artigo apresentado em 29/08/2018

Aprovado em 25/01/2019

Versão final apresentada em 27/01/2019 